\title{
Closed membrane shapes with attached BAR domains subject to external force of actin filaments
}

\author{
Luka Mesarec $^{1 *}$, Wojciech Góźdź ${ }^{2}$, Veronika Kralj Iglič ${ }^{1,3}$, \\ Samo Kralj ${ }^{4,5}$ and Aleš Iglič ${ }^{1,3}$
}

${ }^{1}$ Laboratory of Biophysics, Faculty of Electrical Engineering, University of Ljubljana, Tržaška 25, SI-1000 Ljubljana, Slovenia

${ }^{2}$ Institute of Physical Chemistry, Polish Academy of Sciences, Kasprzaka 44/52, 01-224 Warsaw, Poland

${ }^{3}$ Laboratory of Clinical Biophysics, Faculty of Health Sciences, University of Ljubljana, Zdravstvena 5, SI-1000 Ljubljana, Slovenia

${ }^{4}$ Department of Physics, Faculty of Natural Sciences and Mathematics, University of Maribor, Koroška cesta 160, SI-2000 Maribor, Slovenia

${ }^{5}$ Jožef Stefan Institute, P.O. Box 3000, SI-1000 Ljubljana, Slovenia

${ }^{*}$ Corresponding author. Email address: mesarec.luka@gmail.com

December 31, 2015

The total number of words (including Abstract and figure captions): 6715 The total number of words (not including Abstract and figure captions): 5890 The total number of figures: 8 


\begin{abstract}
Membrane deformations induced by attached BAR superfamily domains could trigger or facilitate the growth of plasma membrane protrusions. The BAR domain family consists of BAR, F-BAR and I-BAR domains, each enforcing a different local curvature when attached to the membrane surface. Our theoretical study mainly focuses on the role of I-BAR in the membrane tubular deformations generated or stabilised by actin filaments. The influence of the area density of membrane attached BAR domains and their intrinsic curvature on the closed membrane shapes (vesicles) was investigated numerically. We derived an analytical approximative expression for the critical relative area density of BARs at which the membrane tubular protrusions on vesicles are most prominent. We have shown that the BARs with a higher intrinsic curvature induce thinner and longer cylindrical protrusions. The average orientation of the membrane attached BARs is altered when the vesicle shape is subjected to external force of growing actin rod-like structure inside a vesicle. The average orientation angle of membrane attached BARs may indicate whether the actin filaments are just stabilising the protrusion or generating it by stretching the vesicle.
\end{abstract}

Keywords: numerical study, biological membranes, membrane proteins, BAR domains, vesicles, actin filaments, membrane tubular deformations, membrane curvature

\title{
1 Introduction
}

The aggregation of proteins in biological membranes plays an important role in normal cell functioning. A disruption in key mechanisms of membrane protein aggregation may lead to different neurodegenerative diseases [1].

The local accumulation and aggregation of membrane proteins may result in the tubulation of cell membrane 224]. The tubulation of cell membrane could be either spontaneous or induced by external agents $[2,5-18]$. The BAR superfamily protein domains are typical substances which change the membrane curvature in such way that the membrane tubes are formed (Fig. 1) [19]. The BAR domains may induce local membrane bending, if the binding energy of the BAR domain to the membrane surface is larger than the energy required to bend the membrane [19]. The BAR domains can dimerise and were suggested to be involved in membrane association 20,21]. Members of the BAR superfamily also play an important role in cell division, cell migration, membrane trafficking and organelle biogenesis 22 . 
The BAR domain superfamily consists of BAR/N-BAR, F-BAR and I-BAR domains, where each of them enforces a different local curvature preference 23 when attached to the membrane surface. The intrinsic shapes of BAR, F-BAR and I-BAR domains and their dimensions are schematically shown in Fig. 1a. Below, we briefly review some key properties of the BAR superfamily members.

The Bin/Amphiphysin/Rvs (BAR) protein domain is a crescent shaped dimer (Fig. 1a) that preferentially binds to negatively charged membranes having a highly positive curvature 20,24,25. BAR domains were found in many different types of proteins [26]. The BAR is a major N-terminal region of homology between amphiphysin relatives [27]. BAR domains are also a part of the Endophilin-A1 protein 28. The BAR domains from endophilin and amphiphysin have hydrophobic amino-acid residues which are inserted into membranes. The amphiphysin BAR dimer is capable of stabilising the membrane curvature 29]. The amphiphysin domains could also tubulate liposomes in vitro like dynamin [30]. The N-BAR domain consists of a BAR domain and an N-terminal amphipathic helix working as a functional unit to generate membrane curvature 31,32. Experiments with lipid bilayer vesicles (liposomes) show that the lateral area density of N-BAR domains on tubes of lipid vesicles is significantly higher compared to the membrane of the connected less curved parent vesicle membrane 33 .

The F-BAR modules have a lower intrinsic curvature than the BAR/N-BAR modules (Fig. 17). The F-BAR modules, present in many proteins, have the preference to bind to membrane surfaces with a positive curvature. F-BAR domains tubulate membranes. The overexpression of the pacsin2 EFC/F-BAR domain can deform liposomes into tubules in vitro and induce cellular microspikes, with the pacsin2 EFC/F-BAR domains concentrated at the neck 34. F-BAR modules are essential for both the microspike formation and the tubulation of membranes [34]. A detailed structure study and the biochemical analysis of FCHo2 F-BAR domain are presented in [35]. F-BARs are frequently linked to cytoskeletal dynamics due to interplay with actin/dynamin [2,4,36].

I-BAR modules (Fig. 1a) preferentially bind to membrane surfaces of a negative curvature [37]. I-BARs are also capable of deforming membranes and inducing plasma membrane protrusions, attaching to the interior of the membrane tubular protrusion, as already discussed above [2, 4]. It was shown that the membrane deforming activity of IRSp53 proteins or I-BAR domains of IRSp53 proteins is essential for the bending of the membrane into filopodia [38, 39]. The structural basis of filopodia formation induced by the IRSp53/MIM homology 
domain (IMD) of human IRSp53 was studied in 40, 41, while IMD/IBAR domains of IRSp53 proteins were recognised and biochemically analysed in 42 . The IMD forms a dimer, with each subunit folded as an antiparallel three-helix bundle. Similar to BAR/F-BAR domains, the IMD/I-BAR domains also bind to the membrane, but the comparison of the structure between the BAR/F-BAR and the IMD/I-BAR domains reveals that they have different membrane curvature preferences. The membrane binding surfaces of the BAR/F-BAR and the IMD/I-BAR modules have opposite curvatures 41]. It was shown that IMDs, when bound to the liposome surface, can induce the formation of membrane tubular invaginations that protrude into the liposome interior 38. The I-BAR domain of IRSp53 (Fig. 17) attached to the inner membrane surface alone may induce dynamic membrane protrusions that lack actin and are thinner than normal filopodia [14].

It is still not fully clear whether the membrane protrusions in the leading edge of migrating cells are pushed and deformed by the polymerising actin filaments inside the cell, or the membrane tubular deformations are only stabilised by actin filaments [2]. Yang et al. suggested $[2$ that actin filaments are not necessary for the generation of the membrane protrusion, but only to stabilise the initial protrusion induced by I-BARs (see also Fig. 6). The formation and maintenance of actin-based cellular protrusions relies on the transport of proteins to the protrusion tip via myosin motors 43 . It was indicated that the formation of initial membrane protrusion may be additionally facilitated by a combined effect of pentasaccharide GM1 and I-BARs [4.

In this paper, we studied the influence of membrane attached BAR domains on the shape of biological membranes. Our theoretical model is constructed in the manner to mimic the impact of the BAR domain superfamily on the tubulation process of the biological membrane or the liposome. We assume that different types of BAR domains, i.e. BAR, F-BAR and I-BAR domains, attach to the membrane surface in a manner as schematically sketched in Fig. 1 1 and not with the opposite side. Therefore, each type of BAR domains enforces a different curvature to the membrane as schematically shown in Fig. 1 1a. In rare cases, BAR domains may also form membrane contact with their side surface, rather than with the concave surface. Such side-laying attachment of the BARs to the membrane surface was first suggested by 25 and later also confirmed by MD simulations at very low values of the local area density of F-BAR domains 44. Our theoretical model is applicable to BAR, F-BAR and I-BAR domains, but our study is mainly focused on the role of I-BAR domains in the formation and stability of the membrane tubular protrusions. We also study the effect of external force, which may be a result of a growing actin 
cytoskeleton inside a vesicle.

The paper is organised as follows. In Section 2, we introduce the theoretical model of the membrane with attached BAR domains and inner actin structure. In Subsection 2.1 and in Supplementary Materials, we describe a general numerical method which is then applied to calculate the equilibrium closed membrane shapes with tubular protrusion and the corresponding lateral distribution of attached BARs which correspond to the minimum of the system free energy. The results of our numerical calculations are presented and discussed in Section 3. The conclusions are presented in Section 4. Finally, the paper is closed by the technical Appendix with the mathematical details about a special case of limit cell/vesicle shapes, which consist of the spherical part and the cylindrical tubular protrusion, where the BAR domains are attached only to the membrane of protrusion.

\section{Model}

We considered a system consisting of flexible rod-like objects attached to a closed membrane. The rod-like objects may represent BAR, FBAR or I-BAR domains, each of them having its own intrinsic curvature. Note that BAR and F-BAR domains usually bind to the outer membrane surfaces, while I-BAR domains attach to the inner surfaces of membranes. The variational parameters of the model are the local membrane curvature $C$ and the local relative area density of BARs $\phi$ which are both determined in the process of variation of the system free energy.

The total free energy of the system, i.e. the free energy of the closed membrane with attached BAR domains, is expressed as an integral of the sum of the densities of membrane bending energy $\left(f_{b}\right)$, BAR domain bending energy $\left(f_{\text {bar }}\right)$, and the configurational entropy of attached BAR domains $\left(f_{m i x}\right)$ :

$$
F_{t o t}=\int_{S}\left(f_{b}+\phi f_{b a r}+f_{\text {mix }}\right) \mathrm{d} S,
$$

where in the second term $f_{b a r}$ the bending energy of the BARs is coupled with the membrane through the local membrane curvature $C$. Here, $\mathrm{d} S$ is an infinitesimal surface element and the integration is carried out over the whole membrane surface area $S$. The free energy density contributions are expressed as

$$
\begin{aligned}
f_{b} & =\frac{\kappa}{2}\left(C_{1}+C_{2}-C_{0}\right)^{2}, \\
f_{\text {bar }} & =\frac{K_{p} L_{0}}{2}\left(C-C_{p}\right)^{2},
\end{aligned}
$$




$$
f_{\text {mix }}=\frac{k_{B} T}{a_{0}}[\phi \ln \phi+(1-\phi) \ln (1-\phi)] .
$$

The membrane bending energy density $f_{b}$ is described within the spontaneous curvature membrane model [45 49], where $\kappa$ is the membrane bending constant, $C_{0}$ is the membrane spontaneous curvature, and $C_{1}$ and $C_{2}$ are the local principal curvatures of the membrane/vesicle surface.

The second part in $F_{\text {tot }}$ (Eq. (3p) is the elastic curvature (bending) energy density of the flexible rod-like BAR domains attached to the membrane. Note that Eq. (3) assumes circular (radial) intrinsic shape of the protein with the constant intrinsic curvature along the long axis of the BAR protein. Moreover, Eq. (3) assumes also that the bending deformation of the BAR domain along its long axis does not substantially vary.

The orientation-dependent energy of a single attached protein domain (Fig. 1b) was originally introduced in Refs. [50,51]. The rigidity and curvature preference of the BAR domain is determined by the flexural rigidity $K_{p}$, the length of the domain $L_{0}$, and the intrinsic (spontaneous) curvature $C_{p}$ of the domain. The local membrane curvature $C$, seen by the attached BAR domain, can be expressed by Euler relation as:

$$
C=H+D \cos (2 \omega) .
$$

Here, $\omega$ is the angle between the normal plane of the first principal curvature $C_{1}$ and the normal plane in which the BAR domain is lying as shown in Fig. 1 $\mathrm{b} ; D=\left(C_{1}-C_{2}\right) / 2$ is the curvature deviator and $H=\left(C_{1}+C_{2}\right) / 2$ the mean curvature at the given location on the membrane surface. The angle $\omega$ corresponding to the minimal $f_{b a r}$, given by Eq. (3), where $C$ is expressed by Eq. (5), equals to

$$
\cos (2 \omega)=\frac{C_{p}-H}{D} .
$$

The configurational entropy contribution of the attached BARs to the free energy is given by Eq. (4), where $k_{B}$ is the Boltzmann constant and $T$ absolute temperature. The symbol $a_{0}$ in Eq. (4) denotes the area of a single attached BAR domain. The role of the entropy increases with decreasing values of $a_{0}$ and vice versa. The local relative area density of BARs $\phi$ ranges between 0 and 1 . Note that Eq. (4) takes into account the finite size (area) of the attached BAR domains (see also $12,52,53]$ ).

The orientational entropy of the BAR domains is related to rotational degree of freedom which depends on the area density of BARs. Namely, at low area density the rotational degree of freedom of the single BAR domain is not sterically hindered by the neighbouring 
BAR domains. To this end all orientational states of single BAR domain are equally probable 6, 11, 17, 51. On the other hand, at higher area densities of BAR domains the rotation of single BAR domain becomes restricted due direct/steric interaction with neighbouring BAR domains, so the configuration of membrane attached elongated rodlike BAR domains may approach to close-packing configuration 44 exhibiting nematic order (see Fig. 5). In this work the curvature dependent contribution of orientational entropy of the BAR domains to the total membrane free energy [51,54] is for the sake of simplicity considered as a constant and therefore omitted in the free energy expression.

Note also, that at very high values of the BAR domain area density the efficiency of the of the membrane curvature variation driven by attached BAR domains start to be diminished because not all BAR domains have the free access to the membrane surface [44]. By using MD simulations it was shown by $\mathrm{Yu}$ and Schulten that F-BAR domains most efficiently generate the membrane curvature variation for the area densities of BAR domains lower than 13 dimers per $1000 \mathrm{~nm}^{2}$ [44.

Fig. 1p shows a special case of the rod-like BAR domain attached to the outer surface of the membrane of cylindrical shape. In general, BARs are attached to the inner or outer surface of the membrane of the arbitrary shape/curvature. Our theoretical description of the energy of BAR domains attached to the membrane surface is general. However, our results are mainly related to I-BAR domains attached to the inner membrane surface.

\section{$2.1 \quad$ Numerical minimisation}

In this work, the vesicle shapes are considered as a closed surface with rotational symmetry. To define the surface of the vesicle, the curve that defines the vesicle profile is rotated around the $z$-axis by the angle $\psi=2 \pi$ (see Fig. S.1 in Suppl. Mat.). To mimic the experimental conditions, the vesicle surface area $S$ and the volume $V$ are kept constant during the minimisation procedure. To study the shapes of the vesicles elongated by an external force, we add the constraint of the constant vertical distance between the poles of the vesicle $z\left(L_{s}\right) \geq d$, where $d$ is the minimum vertical distance 55,56 (Fig. S.1 in Suppl. Mat.).

In order to obtain the equilibrium vesicle shapes, the membrane energy (Eq. (1)) is minimised numerically at non linear constraints for the total volume, the total surface area, the average relative area density of attached BARs, and the fixed vertical distance between the poles of the vesicle [57. We visualise the local area density of 
attached BARs $\phi$ with the color code, as explained in Fig. S.1b in Suppl. Mat.. All lengths are scaled with respect to $R_{0}$, i.e. the radius of the sphere with the same surface area as the surface of the investigated vesicle. Further details of our numerical procedure are described in the Supplementary Materials.

\section{Results and Discussion}

We studied the influence of the relative area density of the membrane attached BAR domains and their intrinsic curvature on the vesicle shapes determined numerically as described in Subsection 2.1. Different examples with and without entropy term in the expression for the membrane free energy are considered. The role of actin filaments in the growth of membrane tubular protrusion is also elucidated.

Firstly, we analysed the influence of the total number of the attached BAR domains on the vesicle shape and the lateral distribution of the attached BARs as presented in Fig. 2. If the entropy of mixing is not taken into account, the membrane attached curved rod-like BAR domains may form tubular protrusions even at a relatively low number of attached BARs. Neglecting the entropic contribution to the membrane free energy, the vesicle protrusion is most prominent at $\phi_{\text {ave }}=0.33$ for the reduced volume $v=0.66$ (Fig. 2 ) and the corresponding vesicle shape is very close to the limit shape, composed of the spherical and the tubular part (Fig. 4). A brief description of a special case of the limit axisymmetric cell/vesicle shapes is given in Appendix A. The calculated vesicle shapes in the lower panel in Fig. 2 were predicted by taking into account the entropy of mixing. One may observe that higher average relative area densities of the attached BARs are required to induce tubular protrusions if the entropic contribution of the attached BARs is taken into account. Namely, the configurational entropic effects hinder the non-homogeneous lateral distribution of the attached BARs. Therefore, at lower values of $\phi_{\text {ave }}$ BARs are practically homogeneously distributed (Fig. 2, lower panel). At higher total numbers of the attached BARs, roughly above $\phi_{\text {ave }} \approx 0.45$, the attached BARs start to accumulate only in one region. Consequently, considering the entropic contribution, the tubular vesicle protrusions appear at $\phi_{\text {ave }} \geq 0.45$ (Fig. 2, lower panel).

The attached BAR domains are predicted to be oriented perpendicularly to the symmetry axis of the tubular protrusion, with the exception of large values of the total number of the attached BAR domains as demonstrated in Fig. 2 (see the calculated shapes for $\phi_{\text {ave }}=0.45$ and 0.65 in the upper panel of the figure). This is in agreement with 
the predictions of MD simulations showing perpendicular orientation of BAR domains with respect to the tube symmetry axis for "circular" (radial) shape of BAR domains 44, i.e. the intrinsic shape of BAR domains with constant intrinsic curvature along the long axis of BAR domain. For non-circular (elliptical) intrinsic shape of BAR domains, where the intrinsic curvature is not constant along the long axis of BAR domain, the results of MD simulations predict small deviation from perpendicular orientation (i.e. tilt) 44.

For high total numbers of the membrane attached BARs there is not enough room for all BARs to be attached only in the region of the membrane protrusion. In such cases, the BAR domains are not only attached to the membrane of the cylindrical part, but also elsewhere on the inner or outer vesicle surface. A new, qualitatively different region is created where the cylindrical protrusion meets the spherical part of the vesicle. In that region, the membrane attached BAR domains are oriented parallel to the symmetry axis of the tubular protrusion. The shapes of the two vesicles on the r.h.s. in the upper panel of Fig. 2 clearly show how the vesicle membrane is locally curved by BARs in order to fit the membrane curvature to their curvature. We predict no similar shapes when the entropy of mixing is taken into account (Fig. 2, lower panel), because the BAR domains are distributed throughout the vesicle surface more homogeneously.

Fig. 3 shows vesicle shapes calculated for different values of the intrinsic curvature of the $\mathrm{BAR}$ domains $C_{p}$. The average relative area density of BARs $\phi_{\text {ave }}$ is the same for all vesicles and it was chosen to be $5 \%$ higher than $\phi_{\min }(\epsilon=0.05$ in Eq. (8)). We analysed the influence of the BAR domain intrinsic curvature on the calculated radius of the vesicle protrusions. Vesicle tubular protrusions are thinner and longer for higher $C_{p}$. The BARs having larger $C_{p}$ prefer to be attached to the membrane of the tubular protrusions with a smaller radius. The calculated tubular protrusions of the vesicles are longer when the radius of the protrusions becomes smaller. Therefore, the surface area of the cylindrical protrusion remains more or less constant during the elongation of the protrusion. The surface area of the vesicle tubular protrusion remains almost constant during elongation, because the total number of the membrane attached BARs is kept constant and the majority of the membrane attached BARs are located on the surface of the membrane which forms the protrusion (Fig. 3).

The radius of filopodia protrusions is primarily influenced by the intrinsic curvature $C_{p}$ of the attached BAR domains, i.e. it is roughly inversely proportional to $C_{p}$. Consequently, for larger magnitudes of $C_{p}$ the membrane tubular protrusions are thinner and longer (Fig. 3). Therefore, in the absence of external force, the membrane attached 
BAR domains define the equilibrium radius of the membrane protrusion. The membrane attached BARs locally curve the membrane in order to minimise the system free energy.

Numerically, we obtained the limit shapes by increasing the spontaneous curvature of the BAR domains $C_{p}$ at a given value of $v$ and $\phi_{\text {ave }}$. When the value of $C_{p}$ is increased, the tubular protrusion becomes thinner and longer (Fig. 3). We can increase the $C_{p}$ until the calculated vesicle shape is composed of a sphere and a tubular protrusion (Fig. 3). Further increasing of $C_{p}$ leads to an almost perfect limit shape (Fig. 3, upper right shape). With the entropy of mixing taken into account, we were not able to predict the perfect limit shape (Fig. 3, lower panel). When the entropy of mixing is included in the minimisation procedure, the BAR domains are distributed more homogeneously and some of them are also found outside the membrane surface of tubular protrusion attached to the membrane of the spheroidal vesicle. For the perfect limit shape, all BARs have to be attached to the membrane surface of the tubular protrusion. We also observed that all the membrane surface attached BARs were oriented in a perpendicular direction to the protrusion's main axis (Fig. 3).

The lower part of the vesicle becomes nearly spherical if $C_{p}$ is large enough as presented in Fig. 3. In this case, the radius of the cylindrical protrusion of the vesicle is very small, compared to the radius of the nearly spherical part of the vesicle: $r<<R$ (see also Fig. 4). If $\phi_{\text {ave }}$ is decreased, it is not possible to obtain the vesicle shapes close to the limit shape, even for very large magnitudes of $C_{p}$. Note that the volume of the cylindrical part of the vesicle compared to the volume of the spherical part is negligible for the vesicle shapes close to the limit shapes. Therefore, the volume of such vesicles is equal to $V \approx 4 \pi R^{3} / 3$ (see also Eq. A.1 in Appendix A). On the other hand, for the nearly limit shapes of the vesicles, the surface area of the vesicle cylindrical protrusion is not negligible in comparison to the surface area of the spherical part of the vesicle, no matter how thin the cylindrical protrusion is.

In general, if $\phi_{\text {ave }}$ (at a given $v$ ) is too low, the vesicle shapes that are similar to the limit shapes cannot be obtained, no matter how much $C_{p}$ is increased, since not enough membrane attached BARs are available to stabilise the entire cylindrical protrusion. By $\phi_{\min }$, we denote the minimal value of the average relative area density of the attached BARs, which is required to obtain the limit shape corresponding to the minimum free energy of the vesicle membrane. By setting $\rho=r / R=0$ in Eq. A.4) (details are given in Appendix A) for the nearly limit vesicle shape the estimated value of the minimum average relative area density $\phi_{\min }$ of the attached rod-like BAR do- 
mains, required to obtain the vesicle shape close to the limit shape of vesicles at a given value of the vesicle reduced volume $v$ is:

$$
\phi_{\min }(v)=\frac{v^{-\frac{2}{3}}-1}{v^{-\frac{2}{3}}} .
$$

At average relative area densities lower than $\phi_{\min }$, the predicted vesicle shapes close to the limit shapes cannot be obtained. The approximate consideration of the limit shapes given in Appendix A is valid, when $\phi_{\text {ave }}$ has the same or slightly higher value than $\phi_{\min }$, i.e.:

$$
\phi_{\text {ave }}(v)=(1+\epsilon) \phi_{\min }(v),
$$

where $\epsilon$ can vary between 0 and approximately 0.1 . For larger values of $\phi_{\text {ave }}$, the approximate purely geometrical consideration of the limit vesicle shapes is not accurate anymore, because the radius of the tubular protrusion $(r)$ is not small enough compared to the radius of the nearly spherical part of the vesicle $(R)$.

Different limit shapes of vesicles for different values of the reduced volume $v$ are presented in Fig. 4. In the calculations, we were increasing the intrinsic curvature of BARs $C_{p}$ for each relative volume of the vesicle until the obtained vesicle shape was very similar to the perfect limit shape. For each value $v$, the average relative area density of BAR domains $\phi_{\text {ave }}(v)$ was determined by Eqs. (7) and (8) for $\epsilon=0.05$.

The values $\phi_{\text {ave }}$ and $v$, which satisfy Eqs. (7) and (8), were inserted into Eqs. A.3 and A.4 in order to calculate the values of $\rho=r / R$ and $\eta=h / R$ (see also Fig. 44), which are both functions of $v$ at the fixed value of $\epsilon$. We present functions $\rho(v)$ and $\eta(v)$ for $\epsilon=$ 0.05 on the left side panels in Fig. 4. It can be seen that $\rho(v)$ is almost a constant function, which means that the radius of the tubular protrusion compared to the radius of the spherical part is practically not changing with the reduced volume. Function $\eta(v)$ is decreasing with increasing $v$, which means that tubular protrusions are becoming shorter.

The calculated shapes in the bottom right panel in Fig. 4 are perfect geometrical limit shapes theoretically predicted from Eqs. A.3 and (A.4) for various values of the reduced volume $v$ and $\phi_{\text {ave }}$, calculated from Eqs. (7) and (8) for $\epsilon=0.05$. The calculated shapes in the upper right hand panel in Fig. 4 result from the numerical minimisation for the same values of $v$ and $\phi_{\text {ave }}$. They are very similar to the perfect limit shapes. The numerically calculated vesicle shapes in the upper right panel in Fig. 4 are in a good agreement with the theoretically predicted shapes in the lower right panel.

It is interesting to examine how the distribution and orientation of the attached BAR domains can be altered by changing the shape of the 
vesicle by an external force. Such force may result from the action of a growing actin cytoskeleton inside the vesicle. The influence of thickness of the inner cytoskeleton rod-like structures on the shape of the membrane protrusion is not considered in this work. We assumed that the membrane protrusion is always thicker than the rod-like structure inside the protrusion. The finite thickness of the cytoskeleton rod-like structure could be simulated by setting the constraint on the minimal radius $r$ of the tubular protrusion.

In Fig. 5 we analysed the calculated vesicle shapes under the influence of an external force. The vesicles were elongated to different vertical lengths $h$. We observed that the orientation of BARs in the tubular protrusion was changing when we increased the cytoskeleton length $d$ (see Fig. 5). The only way for the vesicle to be stretched and at the same time keep the constant value of the reduced volume $v$ is the formation of the tubular protrusion. When the vertical length of the calculated vesicle shape increases, the tubular protrusion becomes thinner and longer. At some critical value of the vesicle vertical length $h$, the first principal curvature of the tubular part of the vesicle $C_{1}$ (see Fig. 1 b) becomes larger than the intrinsic curvature of the BAR domain, $C_{p}$. Starting from that point, the orientation of the attached BARs on the tubular protrusion is adjusted according to Eq. (6) by an appropriate angle, in order to minimise their elastic curvature energy (Eq. (3)). As presented in Fig. 5, above some critical value of vesicle vertical length $(h)$ the angle of the attached BARs orientation $(\omega)$ (defined in Fig. 1 b) starts to become different from zero and continuously increases with the increasing length of the inner cytoskeleton rod-like structure $(d)$ which is equal to the vertical length of the vesicle $(h)$, i.e. $h=d$.

The membrane attached BAR domains laterally oriented for a certain angle $\omega$ which is different from zero may form a chiral surface structure (Fig. 5 and right panel of Fig. 7). Note that chirality is an important phenomenon in many branches of science. For example, the formation of nanotubes may be driven by the self-assembly of chiral amphiphiles 58 60]. Chirality is also important in the formation of thin anisotropic nano strips, which may be transformed into nanotubes 61.

Membrane tubular structures may also be the consequence of anisotropic membrane properties 54,62. Recently, an axisymmetric model of a membrane protrusion that excludes the influence of actin filaments was presented in [12, 63 in order to study the effect of anisotropy of membrane components (prominin nanodomain rafts) and membrane attached molecules (I-BAR domains) on membrane tubulation. It was suggested that the membrane protrusion can be stable even without 
actin filaments 63 in accordance with previous results indicating that the minimization of isotropic bending energy of lipids is not sufficient to explain the stability of the membrane tubular structures [6, 11, 17.

In the process of growing of the plasma membrane protrusion, the initial deformation of the membrane can be generated by I-BAR domains, bound to the interior of a closed membrane, which is schematically shown in Fig. 6. The I-BAR domain intrinsic curvature favours negative membrane curvature of the inner membrane surface, which in turn recruits more I-BAR domains [37] (Fig. 6). I-BAR domains may induce actin self-assembly inside the membrane protrusion and in this way initiate the formation of the membrane protrusions [4]. Actin filaments fill the space inside the membrane protrusion in the process of stochastic polymerisation 2]. The initial membrane deformation during the growth of the membrane protrusion is mechanically stabilised by accumulated I-BAR domains which may also generate the initial growth of the membrane protrusion (filopodic), while actin filaments are self-assembling inside the protrusion (Fig. 6). Actin filaments may not generate the growth of membrane protrusion alone, but they can provide the mechanical support, which is important for the stabilisation of the protrusion during its growth. It seems that actin cytoskeleton is also necessary for a long-term stabilisation of efficient filopodia protrusions 2].

The phase separation of membrane proteins is important in imposing the local membrane curvature, and it may be the driving force for many cellular shape transformations $17,52,64$. The spontaneous phase separation of membrane components (membrane proteins), driven by the forces of actin polymerization and cell-substrate adhesion, was in a quantitative manner predicted for the first time in 64]. Veksler and Gov 64] presented a theoretical model, where the cell membrane contains a constant population of mobile proteins that may form small adhesion complexes. They assumed that some membrane proteins activate actin polymerization. When the cell membrane is stimulated by the external stimulus, membrane bound activators (membrane proteins) are turned on, which causes increased branching and polymerization of the actin cytoskeleton in the vicinity of those proteins. As a result, a local protrusive force starts to act on the membrane 65]. The membrane proteins are very important also in the process of membrane dynamics, which results in the emergence of the complex membrane structures 65 .

The direct interactions between BAR domains are not considered in our model. MD simulations indicate that at high area densities of BAR domains BARs establish side-to-side contact due to charged residues at the lateral contact points (Yu and Schulten, 2013). Side- 
to-side contact may partially originate also from van der Waals forces 66. 67, which are not as strong as Coulombic interactions, but they are always present in biological systems [66. The neighbouring BAR domains may interact only via their terminal groups, mainly due to the electrostatic attraction between the oppositely charged groups 25 . However, the results of MD simulations and experiments predict for F-BAR domains the absence or very weak end-to-end interactions 44. 68 .

The direct interactions between nearest neighbouring BAR domains can be described in most simple way within Bragg-Williams approximation [4,52,54,63,64,69] by considering an additional energy term proportional to $\phi^{2}$. Note, that the Bragg-Williams approximation totally neglects the elongated rod-like shape and orientational ordering of BAR domains. The direct interactions between oriented rod-like BAR domains can be considered also within a linear approximation of the energy coupling with the deviatoric membrane curvature field [67]. In this description, the membrane curvature field that acts on the orientation of BAR domains is created by the BAR domains themselves.

The direct interactions between neighbouring BAR domains at high area densities in close-packing conditions and nematic order regime may be described also by an Ising model 70 . Further, in order to take into account direct interactions one could introduce also a term in the free energy density, which would contain the surface gradient of the BAR domain orientation angle, similar term is used for example in the theory of nematic shells 71 . Such a term would favour the parallel oriented neighbouring $\mathrm{BAR}$ domains.

\section{Conclusions}

The domains of the BAR superfamily, attached to the surface of a closed biological membrane, can in the process of lateral segregation induce the growth of the stable cylindrical membrane protrusions. We have shown that without the application of external force, the membrane attached BAR domains are almost always oriented in a perpendicular direction with respect to the main symmetry axis of tubular membrane protrusion. The exception are the calculated vesicle shapes where the entropic term was not taken into account in the minimisation of free energy and $\phi_{\text {ave }}>>\phi_{\min }$ (see the shapes of the two vesicles on the r.h.s. in the upper panel of Fig. 20.

An external force, for example as a consequence of the growing actin rod-like structure inside the vesicle, may change the average ori- 
entation of the rod-like membrane attached BAR domains as schematically shown in Fig. 7. When the vesicle is stretched by the external force the cylindrical membrane protrusion becomes not only longer but also thinner (regardless of the value of $C_{p}$ ) in order to keep the constant volume of the vesicle. In this case the membrane attached BARs adjust their lateral orientation in order to minimise the system free energy. Accordingly, we have predicted that the membrane attached BARs are rotated by an angle $\omega$ different from zero (see Fig. 1 b) when the external force is acting on the vesicle membrane as schematically shown in Fig. 7. Above a certain critical value of the vesicle vertical length enforced by the inner actin rod-like structure, the orientation angle of the attached BARs $\omega$ is continuously increasing with the length of the actin rod-like structure, i.e. with the vertical length of the vesicle.

In the future, it would be interesting to experimentally determine the lateral orientation of the BAR domains attached to the inner or outer surface of the vesicle or cell membrane protrusions, when external force acting on the membrane is present. External force could be applied experimentally or it may be a result of the action of a growing cytoskeleton inside of the vesicle or cell. External force can be experimentally applied to the surface of the cell membrane by cantilever of the atomic force microscope 72 .

To conclude, the lateral orientation of the membrane attached BARs may be a consequence of the force created by actin cytoskeleton. If the orientation of the membrane attached BAR domains were different from $\omega=0$ (see also Fig. 1 b) in the absence of the experimentally induced external force, this would mean that the role of the actin cytoskeleton is not only to mechanically stabilise the membrane protrusion, but also to stretch it, i.e. to induce the process of the protrusion growth. The phenomenon of laterally oriented membrane attached BAR domains might be important also for intercellular communication. Namely, the membrane structures like membrane tubular protrusions or tubular membrane vesicles, with the attached BAR domains oriented by a similar angle $\omega$ might have a higher tendency to communicate with each other, due to the similar topological properties of the tops of the membrane tubular protrusions.

\section{A Limit shapes}

Here, we briefly describe the limit axisymmetric cell/vesicle shapes consisting of the spherical part and cylindrical tubular protrusion as presented in Fig. 4. We assume that the attached BAR domains are 
homogeneously distributed on the surface of the cylindrical part only, while the surface of the spherical part is completely free of the attached BARs. We assume also that the cylindrical protrusion is very thin, compared to the radius of the spherical part $(r<<R)$. In the following, we shall determine the quantitative geometrical characteristic of the above described limit shapes and conditions.

The volume $V$ and the surface area $A$ of the limit shape equals to

$$
\begin{aligned}
V & =\frac{4}{3} \pi R^{3}+\pi r^{2} h, \\
A & =4 \pi R^{2}+2 \pi r h,
\end{aligned}
$$

where $R, h$ and $r$ are defined in the inset in the upper left panel in Fig. 4. The average relative area density of the attached BAR domains equals to

$$
\phi_{\text {ave }}=\frac{2 \pi r h}{4 \pi R^{2}+2 \pi r h}=\frac{1}{1+\frac{2}{\rho \eta}},
$$

where $\rho=r / R$ and $\eta=h / R$. An important geometrical parameter defining the vesicle shape is its reduced volume $v$. It is defined as the ratio of the vesicle volume $V$ and the volume of the sphere $V_{0}=$ $4 \pi R_{0}^{3} / 3$ with the same surface area:

$$
v=\frac{V}{V_{0}}=\frac{1+\frac{3}{4} \rho^{2} \eta}{\left(1+\frac{\rho \eta}{2}\right)^{3 / 2}}=\frac{1+\frac{3}{2} \frac{\rho}{\phi_{\text {ave }}^{-1}-1}}{\left(1+\frac{1}{\phi_{\text {ave }}^{-1}-1}\right)^{3 / 2}},
$$

where we took into account $4 \pi R_{0}^{2}=4 \pi R^{2}+2 \pi r h$ and $\rho \eta$ was expressed from Eq. A.3) as a function of $\phi_{\text {ave }}$. Eqs. (A.4) and (A.3) allow us to determine the relative radius of the cylindrical protrusion $\rho$ and its relative height $\eta$ as functions of the reduced volume $v$ and $\phi_{\text {ave }}$.

\section{Acknowledgement}

We would like to acknowledge the support from NCN grant No 2012/05/B/ST3/03302 and the grant of the Slovenian Research Agency (ARRS) No. P-0232. Dr. N. Bobrovska Dr. is gratefully acknowledged for help and useful discussions.

\section{References}

[1] A. M. Morris, M. A. Watzky, and R. G. Finke, "Protein aggregation kinetics, mechanism, and curve-fitting: a review of the 
literature," Biochimica et Biophysica Acta (BBA)-Proteins and Proteomics, vol. 1794, no. 3, pp. 375-397, 2009.

[2] C. Yang, M. Hoelzle, A. Disanza, G. Scita, and T. Svitkina, "Coordination of membrane and actin cytoskeleton dynamics during filopodia protrusion," PloS one, vol. 4, no. 5, p. e5678, 2009.

[3] H. Zhao, A. Pykäläinen, and P. Lappalainen, "I-bar domain proteins: linking actin and plasma membrane dynamics," Current opinion in cell biology, vol. 23, no. 1, pp. 14-21, 2011.

[4] D. Kabaso, M. Lokar, V. Kralj-Iglič, P. Veranič, and A. Iglič, "Temperature and cholera toxin $\mathrm{b}$ are factors that influence formation of membrane nanotubes in rt4 and t24 urothelial cancer cell lines," Int J Nanomedicine, vol. 6, pp. 495-509, 2011.

[5] I. Tsafrir, Y. Caspi, M.-A. Guedeau-Boudeville, T. Arzi, and J. Stavans, "Budding and tubulation in highly oblate vesicles by anchored amphiphilic molecules," Phys. Rev. Lett., vol. 91, p. 138102, 2003.

[6] V. Kralj-Iglič, A. Iglič, H. Hägerstrand, and P. Peterlin, "Stable tubular microvesicles of the erythrocyte membrane induced by dimeric amphiphiles," Phys. Rev. E, vol. 61, pp. 4230 - 4234, 2000 .

[7] G. Koster, M. VanDuijn, B. Hofs, and M. Dogterom, "Membrane tube formation from giant vesicles by dyanmic association of motor proteins," PNAS, vol. 100, pp. $15583-15588,2003$.

[8] A. Roux, D. Cuvelier, P. Nassoy, J. Prost, P. Bassereau, and B. Goud, "Role of curvature and phase transition in lipid sorting and fission of membrane tubules," EMBO J., vol. 24, pp. 1537 $1545,2005$.

[9] W. Huttner and A. Schmidt, "Membrane curvature: a case of endofilin," Trends Cell Biol., vol. 12, pp. 155 - 158, 2002.

[10] D. Davis and S. Sowinski, "Membrane nanotubes: dynamic longdistance connections between animal cells," Nat. Rev. Mol. Cell Bio., vol. 9, pp. $431-436,2008$.

[11] V. Kralj-Iglič, A. Iglič, G. Gomišček, F. Sevšek, V. Arrigler, and H. Hagerstrand, "Microtubes and nanotubes of a phospholipid bilayer membrane," Journal of Physics A: Mathematical and General, vol. 35, no. 7, pp. 1533-1549, 2002.

[12] N. Bobrovska, W. Góźdź, V. Kralj-Iglič, and A. Iglič, "On the Role of Anisotropy of Membrane Components in Formation and Stabilization of Tubular Structures in Multicomponent Membranes," PLOS ONE, vol. 8, p. e73941, SEP 162013. 
[13] P. Veranič, M. Lokar, G. J. Schütz, J. Weghuber, S. Wieser, H. Hägerstrand, V. Kralj-Iglič, and A. Iglič, "Different types of cell-to-cell connections mediated by nanotubular structures," Biophysical journal, vol. 95, no. 9, pp. 4416-4425, 2008.

[14] K. B. Lim, W. Bu, W. I. Goh, E. Koh, S. H. Ong, T. Pawson, T. Sudhaharan, and S. Ahmed, "The cdc42 effector irsp53 generates filopodia by coupling membrane protrusion with actin dynamics," Journal of Biological Chemistry, vol. 283, no. 29, pp. 20454-20472, 2008.

[15] A. Tian and T. Baumgart, "Sorting of lipids and proteins in membrane curvature gradients," Biophysical Journal, vol. 96, no. 7, pp. 2676-2688, 2009.

[16] J. Hurtig, D. T. Chiu, and B. Önfelt, "Intercellular nanotubes: insights from imaging studies and beyond," Wiley Interdisciplinary Reviews: Nanomedicine and Nanobiotechnology, vol. 2, no. 3, pp. 260-276, 2010.

[17] A. Iglič, M. Lokar, B. Babnik, T. Slivnik, P. Veranič, H. Hägerstrand, and V. Kralj-Iglič, "Possible role of flexible red blood cell membrane nanodomains in the growth and stability of membrane nanotubes," Blood Cells, Molecules, and Diseases, vol. 39, no. 1, pp. 14-23, 2007.

[18] R. Shlomovitz, N. Gov, and A. Roux, "Membrane-mediated interactions and the dynamics of dynamin oligomers on membrane tubes," New Journal of Physics, vol. 13, no. 6, p. 065008, 2011.

[19] J. Zimmerberg and S. McLaughlin, "Membrane curvature: how bar domains bend bilayers," Current Biology, vol. 14, no. 6, pp. R250-R252, 2004.

[20] B. J. Peter, H. M. Kent, I. G. Mills, Y. Vallis, P. J. G. Butler, P. R. Evans, and H. T. McMahon, "Bar domains as sensors of membrane curvature: the amphiphysin bar structure," Science, vol. 303, no. 5657, pp. 495-499, 2004.

[21] M. M. Kessels and B. Qualmann, "Syndapin oligomers interconnect the machineries for endocytic vesicle formation and actin polymerization," Journal of Biological Chemistry, vol. 281, no. 19, pp. 13285-13299, 2006.

[22] A. Frost, V. M. Unger, and P. De Camilli, "The bar domain superfamily: membrane-molding macromolecules," Cell, vol. 137, no. 2, pp. 191-196, 2009.

[23] S. Suetsugu, "The proposed functions of membrane curvatures mediated by the bar domain superfamily proteins," Journal of biochemistry, vol. 148, no. 1, pp. 1-12, 2010. 
[24] H. J. Chial, P. Lenart, and Y. Q. Chen, "Appl proteins fret at the bar: direct observation of appl1 and appl2 bar domain-mediated interactions on cell membranes using fret microscopy," PloS one, vol. 5, no. 8, p. e12471, 2010.

[25] D. Kabaso, E. Gongadze, P. Elter, U. Van Rienen, J. Gimsa, V. Kralj-Iglič, and A. Iglič, "Attachment of rod-like (bar) proteins and membrane shape," Mini reviews in medicinal chemistry, vol. 11, no. 4, pp. 272-282, 2011.

[26] C. Tarricone, B. Xiao, N. Justin, P. Walker, K. Rittinger, S. Gamblin, and S. Smerdon, "The structural basis of arfaptin-mediated cross-talk between rac and arf signalling pathways," Nature, vol. 411, no. 6834, pp. 215-219, 2001.

[27] D. Sakamuro, K. J. Elliott, R. Wechsler-Reya, and G. C. Prendergast, "Bin1 is a novel myc-interacting protein with features of a tumour suppressor," Nature genetics, vol. 14, no. 1, pp. 69-77, 1996.

[28] W. Weissenhorn, "Crystal structure of the endophilin-a1 bar domain," Journal of molecular biology, vol. 351, no. 3, pp. 653-661, 2005.

[29] G. Khelashvili, D. Harries, and H. Weinstein, "Modeling membrane deformations and lipid demixing upon protein-membrane interaction: the bar dimer adsorption," Biophysical journal, vol. 97, no. 6, pp. 1626-1635, 2009.

[30] K. Takei, V. I. Slepnev, V. Haucke, and P. De Camilli, "Functional partnership between amphiphysin and dynamin in clathrinmediated endocytosis," Nature cell biology, vol. 1, no. 1, pp. 3339, 1999.

[31] J. L. Gallop, C. C. Jao, H. M. Kent, P. J. G. Butler, P. R. Evans, R. Langen, and H. T. McMahon, "Mechanism of endophilin nbar domain-mediated membrane curvature," The EMBO journal, vol. 25, no. 12, pp. 2898-2910, 2006.

[32] M. Masuda, S. Takeda, M. Sone, T. Ohki, H. Mori, Y. Kamioka, and N. Mochizuki, "Endophilin bar domain drives membrane curvature by two newly identified structure-based mechanisms," The EMBO Journal, vol. 25, no. 12, pp. 2889-2897, 2006.

[33] M. C. Heinrich, B. R. Capraro, A. Tian, J. M. Isas, R. Langen, and T. Baumgart, "Quantifying membrane curvature generation of drosophila amphiphysin n-bar domains," The journal of physical chemistry letters, vol. 1, no. 23, pp. 3401-3406, 2010.

[34] A. Shimada, K. Takano, M. Shirouzu, K. Hanawa-Suetsugu, T. Terada, K. Toyooka, T. Umehara, M. Yamamoto, 
S. Yokoyama, and S. Suetsugu, "Mapping of the basic aminoacid residues responsible for tubulation and cellular protrusion by the efc/f-bar domain of pacsin2/syndapin ii," FEBS letters, vol. 584, no. 6, pp. 1111-1118, 2010.

[35] W. M. Henne, H. M. Kent, M. G. Ford, B. G. Hegde, O. Daumke, P. J. G. Butler, R. Mittal, R. Langen, P. R. Evans, and H. T. McMahon, "Structure and analysis of fcho2 f-bar domain: a dimerizing and membrane recruitment module that effects membrane curvature," Structure, vol. 15, no. 7, pp. 839-852, 2007.

[36] T. Itoh, K. S. Erdmann, A. Roux, B. Habermann, H. Werner, and P. De Camilli, "Dynamin and the actin cytoskeleton cooperatively regulate plasma membrane invagination by bar and f-bar proteins," Developmental cell, vol. 9, no. 6, pp. 791-804, 2005.

[37] C. Prévost, H. Zhao, J. Manzi, E. Lemichez, P. Lappalainen, A. Callan-Jones, and P. Bassereau, "Irsp53 senses negative membrane curvature and phase separates along membrane tubules," Nature communications, vol. 6, 2015.

[38] P. K. Mattila, A. Pykäläinen, J. Saarikangas, V. O. Paavilainen, H. Vihinen, E. Jokitalo, and P. Lappalainen, "Missing-inmetastasis and irsp53 deform pi $(4,5)$ p2-rich membranes by an inverse bar domain-like mechanism," The Journal of cell biology, vol. 176 , no. 7 , pp. 953-964, 2007.

[39] S. Krugmann, I. Jordens, K. Gevaert, M. Driessens, J. Vandekerckhove, and A. Hall, "Cdc42 induces filopodia by promoting the formation of an irsp53: Mena complex," Current Biology, vol. 11, no. 21 , pp. $1645-1655,2001$.

[40] T. H. Millard, G. Bompard, M. Y. Heung, T. R. Dafforn, D. J. Scott, L. M. Machesky, and K. Fütterer, "Structural basis of filopodia formation induced by the irsp $53 / \mathrm{mim}$ homology domain of human irsp53," The EMBO journal, vol. 24, no. 2, pp. 240-250, 2005 .

[41] S. H. Lee, F. Kerff, D. Chereau, F. Ferron, A. Klug, and R. Dominguez, "Structural basis for the actin-binding function of missing-in-metastasis," Structure, vol. 15, no. 2, pp. 145-155, 2007.

[42] A. Yamagishi, M. Masuda, T. Ohki, H. Onishi, and N. Mochizuki, "A novel actin bundling/filopodium-forming domain conserved in insulin receptor tyrosine kinase substrate p53 and missing in metastasis protein," Journal of Biological Chemistry, vol. 279, no. 15 , pp. 14929-14936, 2004. 
[43] A. Yochelis, S. Ebrahim, B. Millis, R. Cui, B. Kachar, M. Naoz, and N. S. Gov, "Self-organization of waves and pulse trains by molecular motors in cellular protrusions," Scientific reports, vol. $5,2015$.

[44] H. Yu and K. Schulten, "Membrane sculpting by f-bar domains studied by molecular dynamics simulations," PLoS Comput Biol, vol. 9, no. 1, p. e1002892, 2013.

[45] H. J. Deuling and W. Helfrich, "Red blood cell shapes as explained on the basis of curvature elasticity," Biophys J., vol. 16, pp. 861-868, 1976.

[46] W. Helfrich, "Elastic properties of lipid bilayers: theory and possible experiments," Z. Naturforsch., vol. 28, pp. 693-703, 1973.

[47] E. A. Evans, "Bending resistance and chemically induced moments in membrane bilayers," Biophys. J., vol. 14, pp. 923-931, 1974.

[48] Z. Shi and T. Baumgart, "Dynamics and instabilities of lipid bilayer membrane shapes," Advances in colloid and interface science, vol. 208, pp. 76-88, 2014.

[49] A. Boulbitch, R. Simson, D. Simson, R. Merkel, W. Häckl, M. Bärmann, and E. Sackmann, "Shape instability of a biomembrane driven by a local softening of the underlying actin cortex," Physical review E, vol. 62, no. 3, p. 3974, 2000.

[50] A. Iglič, T. Slivnik, and V. Kralj-Iglič, "Elastic properties of biological membranes influenced by attached proteins," Journal of biomechanics, vol. 40, no. 11, pp. 2492-2500, 2007.

[51] Š. Perutková, V. Kralj-Iglič, M. Frank, and A. Iglič, "Mechanical stability of membrane nanotubular protrusions influenced by attachment of flexible rod-like proteins," Journal of biomechanics, vol. 43, no. 8, pp. 1612-1617, 2010.

[52] H. Hägerstrand, L. Mrowczynska, U. Salzer, R. Prohaska, K. A. Michelsen, V. Kralj-Iglič, and A. Iglič, "Curvature-dependent lateral distribution of raft markers in the human erythrocyte membrane," Molecular membrane biology, vol. 23, no. 3, pp. 277-288, 2006.

[53] M. Fošnarič, A. Iglič, T. Slivnik, and V. Kralj-Iglič, "Flexible membrane inclusions and membrane inclusions induced by rigid globular proteins," Advances in planar lipid bilayers and liposomes, vol. 7, pp. 143-168, 2008.

[54] A. Iglič, V. Kralj-Iglič, and D. Drobne, Nanostructures in Biological Systems: Theory and Applications. Pan Stanford Publishing Pte. Ltd., 2015. 
[55] W. T. Góźdź, "Spontaneous curvature induced shape transformation of tubular polymersomes," Langmuir, vol. 20, pp. 7385-7391, 2004.

[56] W. T. Góźdź, N. Bobrovska, and A. Ciach, "Separation of components in lipid membranes induced by shape transformation," J. Chem. Phys., vol. 137, no. 1, p. 015101, 2012.

[57] W. T. Góźdź, "The interface width of separated two-component lipid membranes," J. Phys. Chem. B, vol. 110, pp. 21981-21986, 2006.

[58] L. Ziserman, H.-Y. Lee, S. R. Raghavan, A. Mor, and D. Danino, "Unraveling the mechanism of nanotube formation by chiral selfassembly of amphiphiles," Journal of the American Chemical Society, vol. 133, no. 8, pp. 2511-2517, 2011.

[59] L. Ziserman, A. Mor, D. Harries, and D. Danino, "Curvature instability in a chiral amphiphile self-assembly," Physical review letters, vol. 106, no. 23, p. 238105, 2011.

[60] D. Danino, "Cryo-tem of soft molecular assemblies," Current Opinion in Colloid \& Interface Science, vol. 17, no. 6, pp. 316329, 2012.

[61] A. Iglič, M. Tzaphlidou, M. Remškar, B. Babnik, M. Daniel, and V. Kralj-Iglič, "Stable shapes of thin anisotropic nano-strips," Fullerenes, Nanotubes, and Carbon Nonstructures, vol. 13, no. 3, pp. 183-192, 2005.

[62] A. Iglič, B. Babnik, U. Gimsa, and V. Kralj-Iglič, "On the role of membrane anisotropy in the beading transition of undulated tubular membrane structures," Journal of Physics A: Mathematical and General, vol. 38, no. 40, p. 8527, 2005.

[63] D. Kabaso, N. Bobrovska, W. Góźdź, N. Gov, V. Kralj-Iglič, P. Veranič, and A. Iglič, "On the role of membrane anisotropy and bar proteins in the stability of tubular membrane structures," Journal of biomechanics, vol. 45, no. 2, pp. 231-238, 2012.

[64] A. Veksler and N. S. Gov, "Phase transitions of the coupled membrane-cytoskeleton modify cellular shape," Biophysical journal, vol. 93, no. 11, pp. 3798-3810, 2007.

[65] N. S. Gov and A. Gopinathan, "Dynamics of membranes driven by actin polymerization," Biophysical journal, vol. 90, no. 2, pp. 454-469, 2006.

[66] J. N. Israelachvili, Intermolecular and surface forces: revised third edition. Academic press, 2011. 
[67] V. Kralj-Iglič, B. Babnik, D. R. Gauger, S. May, and A. Iglič, "Quadrupolar ordering of phospholipid molecules in narrow necks of phospholipid vesicles," Journal of statistical physics, vol. 125, no. 3, pp. 727-752, 2006.

[68] A. Frost, R. Perera, A. Roux, K. Spasov, O. Destaing, E. H. Egelman, P. De Camilli, and V. M. Unger, "Structural basis of membrane invagination by f-bar domains," Cell, vol. 132, no. 5 , pp. 807-817, 2008.

[69] T. L. Hill, An introduction to statistical thermodynamics. Dover Press, 1986.

[70] E. Ising, "Beitrag zur theorie des ferromagnetismus," Zeitschrift für Physik A Hadrons and Nuclei, vol. 31, no. 1, pp. 253-258, 1925.

[71] S. Kralj, R. Rosso, and E. G. Virga, "Curvature control of valence on nematic shells," Soft Matter, vol. 7, pp. 670-683, 2011.

[72] A. Boulbitch, "Deflection of a cell membrane under application of a local force," Physical Review E, vol. 57, no. 2, p. 2123, 1998.

[73] "Bar superfamily home page." http://bar-superfamily.org/. Accessed: 2015-03-17. 
(a)

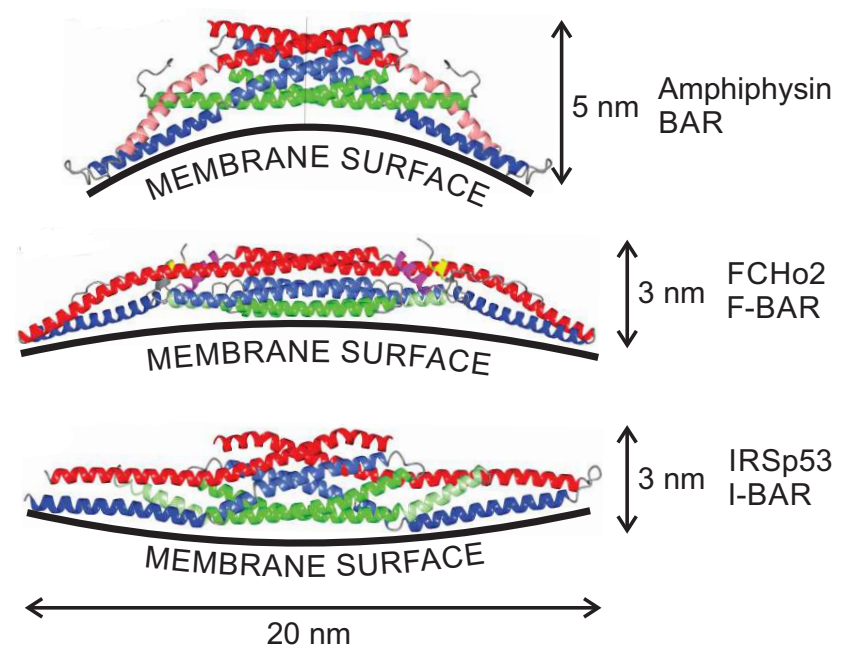

(b)

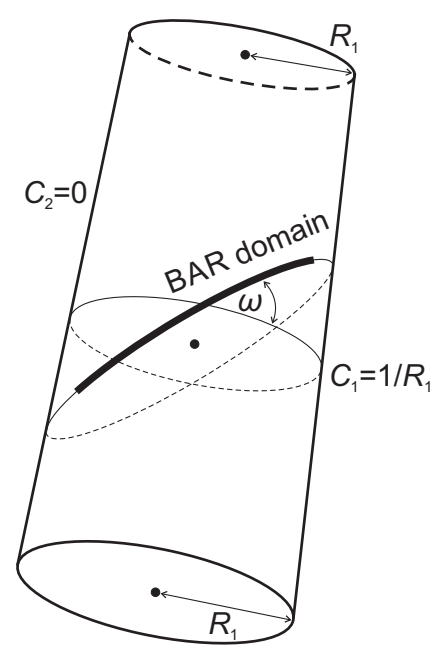

Figure 1: Schematic presentations of the BAR superfamily domains and the cylindrical surface with the attached rod-like BAR domain. (a) The BAR domains are presented with their typical dimensions and curvature preferences (partially adapted from [73]). (b) The flexible rod-like BAR domain attached to the membrane surface of the cylindrical shape, where $R_{1}$ is the radius of a cylinder. Angle $\omega$ is the angle between the normal plane of the first principal curvature $C_{1}$ and the normal plane in which the BAR domain is lying. $C_{2}$ is the second principal curvature. 


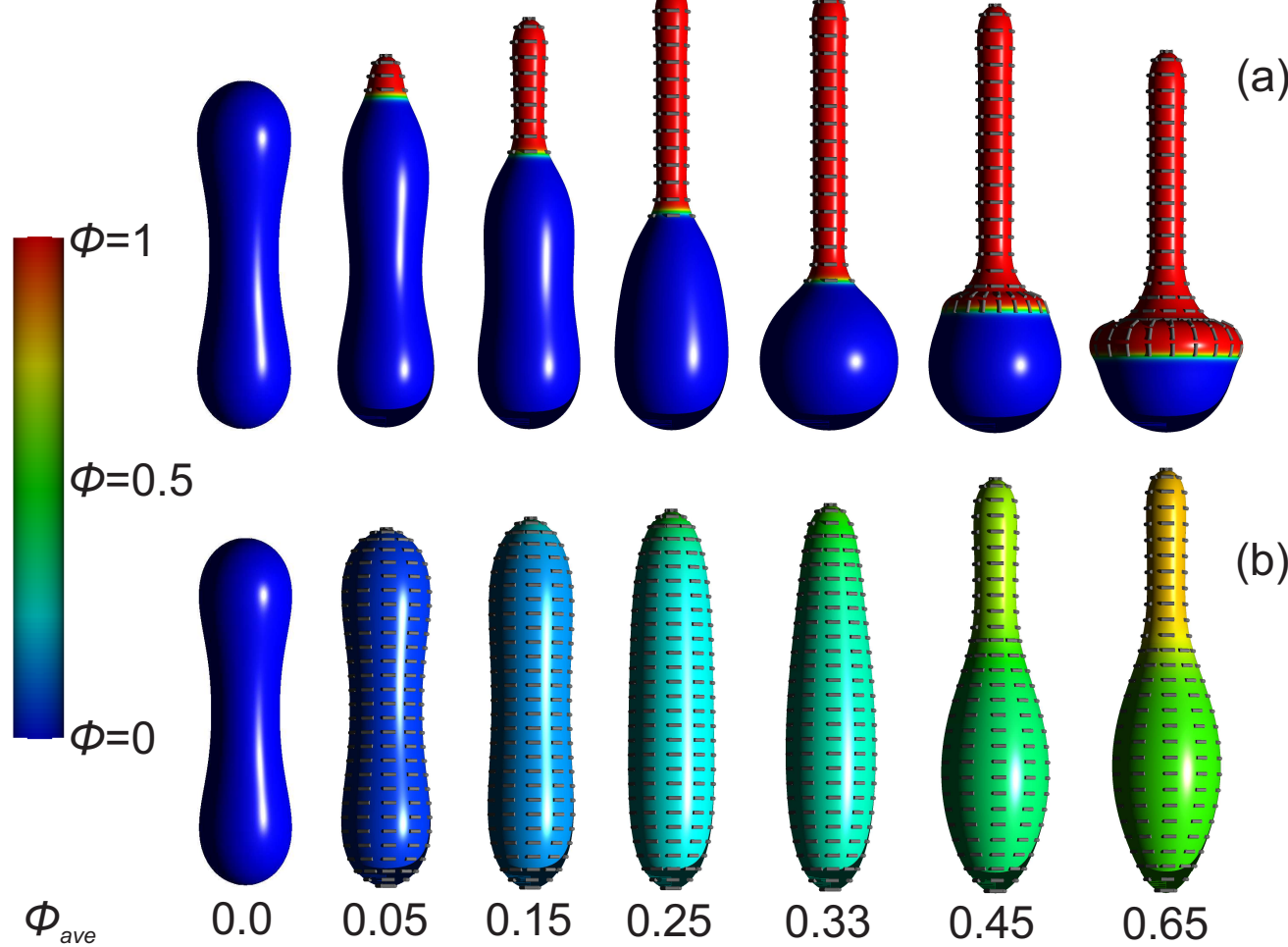

a)

b)

Figure 2: Vesicle shapes calculated for different values of the average relative area density of BARs $\phi_{\text {ave }}$. The directions of BARs are denoted by grey lines. The local relative area density of attached BARs $\phi$ is visualised with the color code. The shapes were calculated for the following parameters: $C_{0}=0, v=0.66, C_{p}=8, K_{p} L_{0} / 2=\kappa$. The shapes in the upper panel were calculated without the entropy of mixing. The entropy of mixing was taken into account for the shapes in the lower panel: $\kappa=30 k_{B} T, R_{0}=250 \mathrm{~nm}$, $a_{0}=100 \mathrm{~nm}^{2}$. 

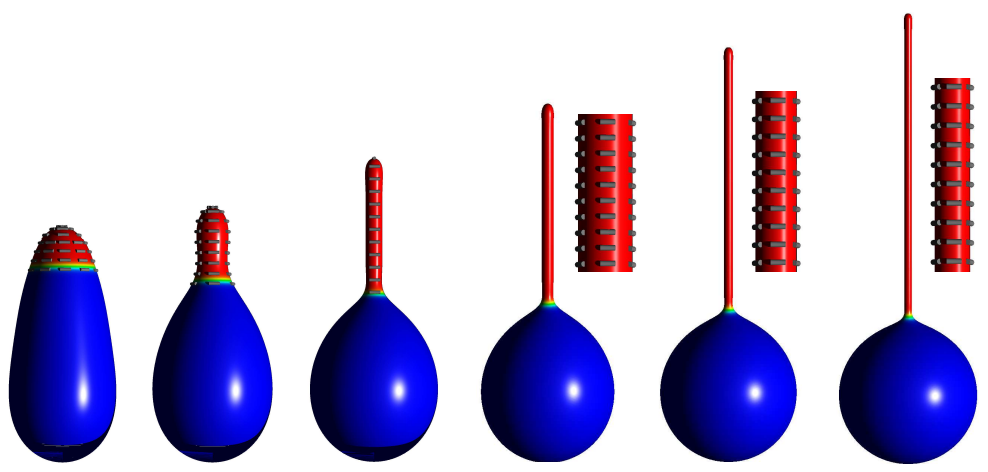

$C_{p}$

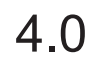

7.0

13.0

19.0
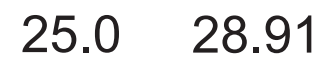

$\Phi=0.5$
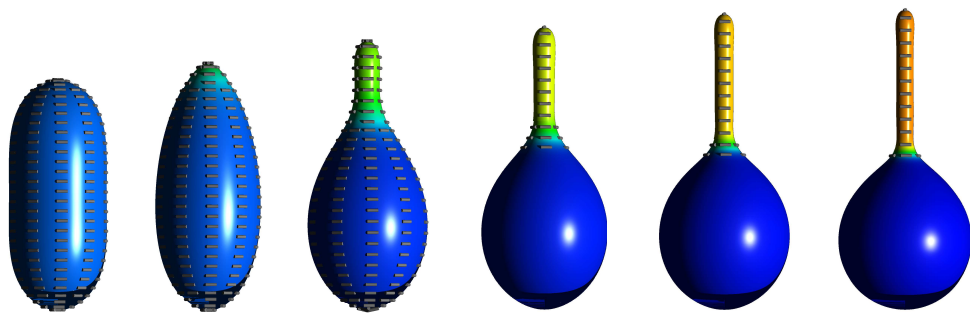

$C_{p} \quad 4.0$

10.0

11.0

12.0

$13.0 \quad 13.75$

Figure 3: Vesicle shapes, calculated for different values of $C_{p}$ (curvature of the BAR domains). The directions of BARs are denoted by grey lines. The local relative area density of attached BARs $\phi$ is visualised with the color code. The entropy of mixing was taken into account only for the shapes in the lower panel. Parameters: $C_{0}=0, v=0.85, \phi_{\text {ave }}=0.1078$, $K_{p} L_{0} / 2=\kappa, \kappa=30 k_{B} T, R_{0}=250 \mathrm{~nm}, a_{0}=100 \mathrm{~nm}^{2}$. 

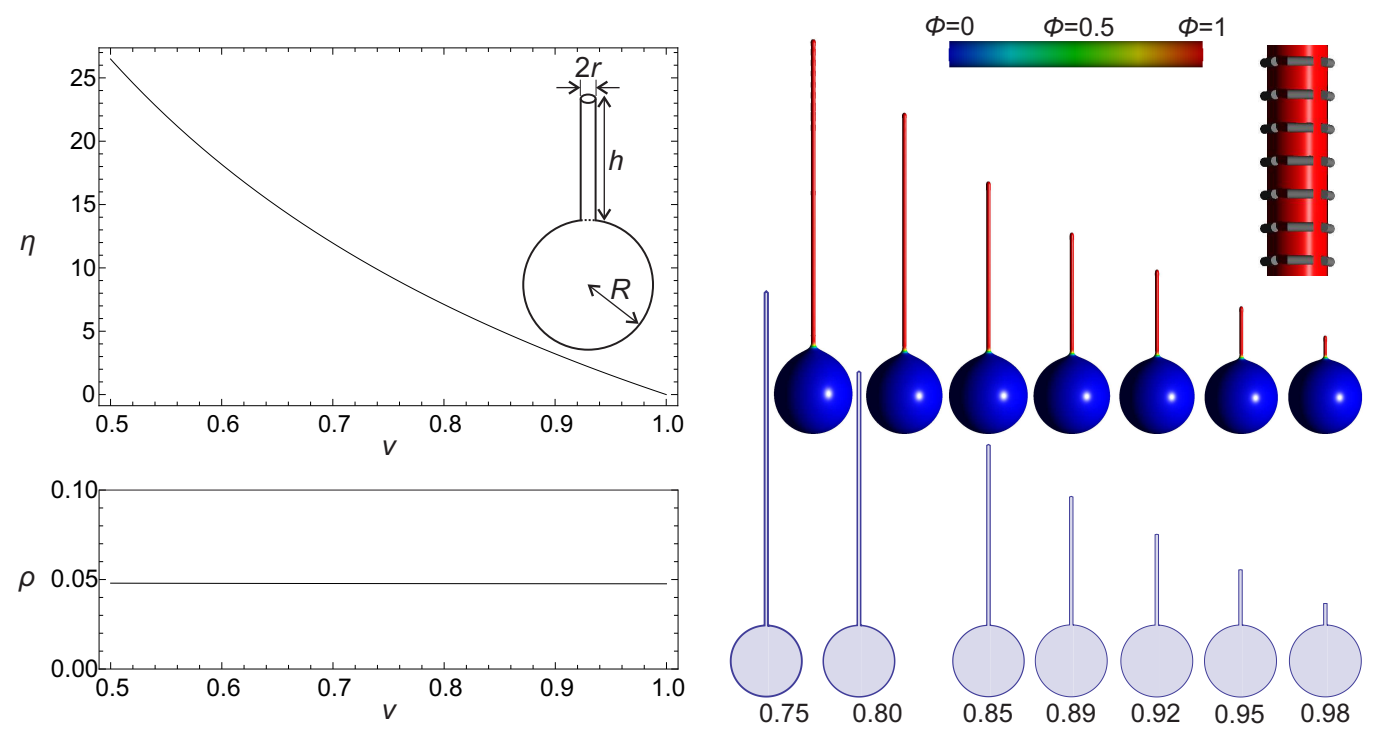

Figure 4: Dimensionless parameters $\eta=h / R$ and $\rho=r / R$ with the corresponding limit shaped vesicles as the functions of the reduced volume $v$ for $\epsilon=0.05$. The inset in the upper left panel represents limit vesicle shape, composed of the spherical part with radius $R$ and the cylindrical protrusion of the vertical length $h$ and the radius $r$. The shapes in the bottom right panel are perfect geometric limit shapes calculated for different values of the reduced volume for $\epsilon=0.05$. The shapes in the upper right panel were minimised without the entropy of mixing for: $\epsilon=0.05, C_{0}=0$, $K_{p} L_{0} / 2=\kappa$, where the local relative area density of attached BARs $\phi$ is visualised with the color code. $C_{p}$ was increased until the vesicle shapes close to the perfect limit shapes were reached. All BARs are oriented perpendicularly to the protrusions main axis as schematically shown in the upper right corner. 


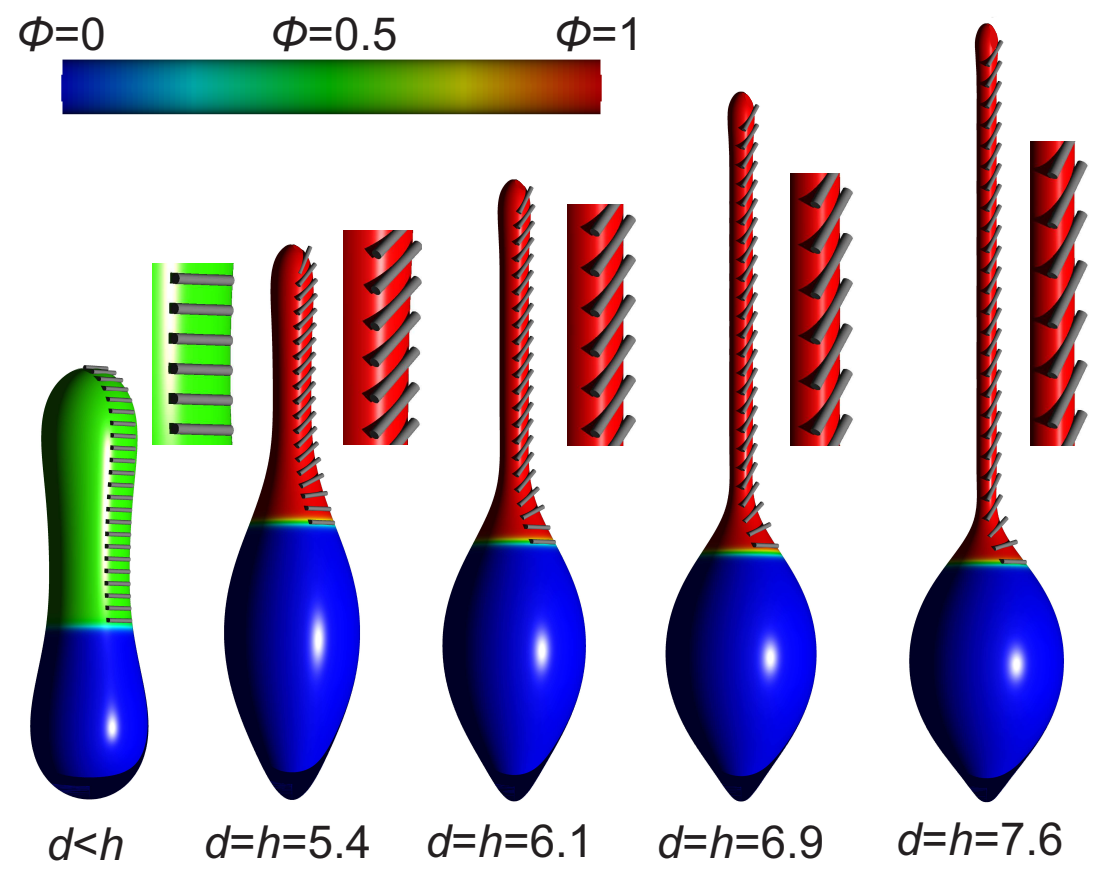

Figure 5: Equilibrium vesicle shapes calculated for different lengths of the actin cytoskeleton rod-like structure inside the vesicle. We denote by $d$ the cytoskeleton length, by $h$ the vertical height of the vesicle, and by $\phi$ the local relative area density of attached BARs, which is visualised with the color code. The directions of the orientation of BARs are denoted by grey lines. The entropy of mixing was not taken into account. Model parameters are: $C_{0}=0, v=0.65, \phi_{\text {ave }}=0.30, C_{p}=3.0, K_{p} L_{0} / 2=\kappa$. 


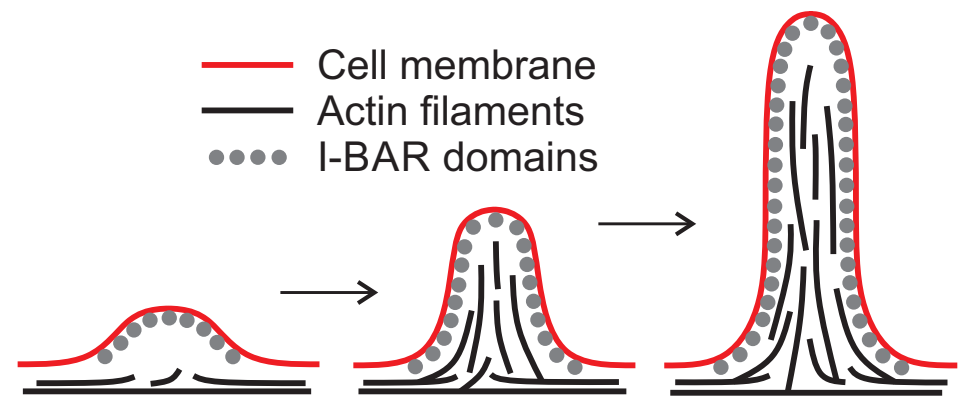

Figure 6: Schematic presentation of the possible mechanism of the growth of the membrane protrusion. I-BAR domains, bound to the inner membrane surface, may generate the initial plasma membrane outward deformation. The space inside of the deformation/membrane protrusion is filled with the actin filaments. The I-BAR domains start to accumulate inside the tubular protrusion because of the favourable negative membrane curvature. I-BAR domains generated cylindrical filopodia protrusions are stabilised by actin self-assembly inside of the protrusion.

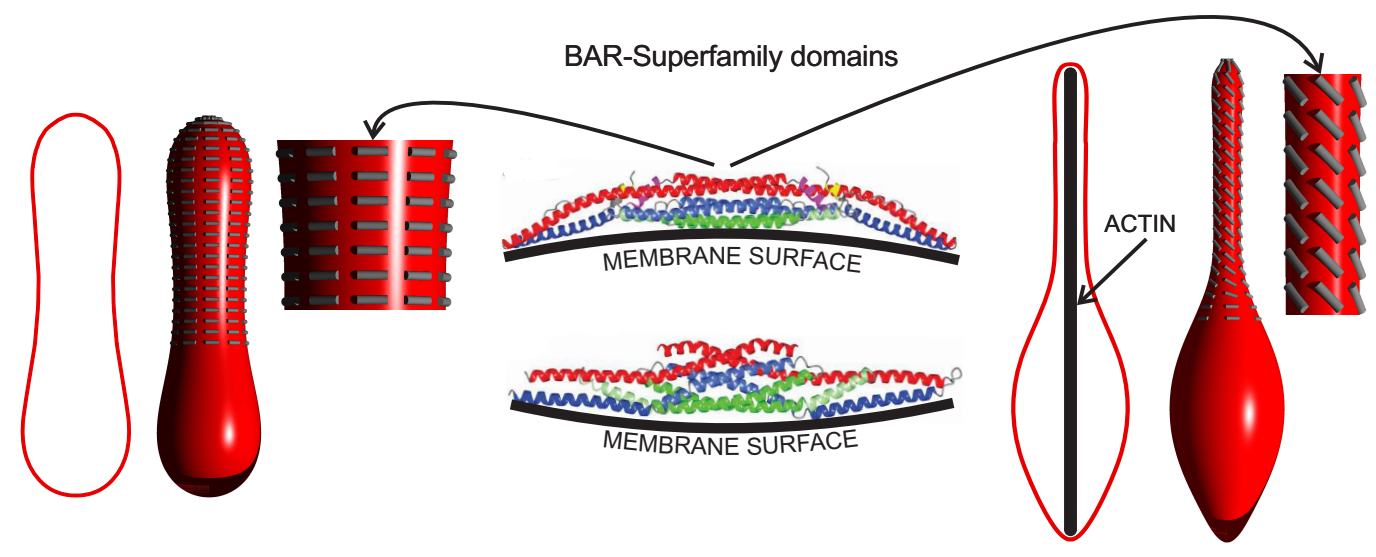

Figure 7: The impact of external force on the orientation of BAR domains. The lateral orientation of the membrane attached BAR domains (grey lines) is changed, when the vesicle is elongated by external force, which may result from the action of a growing cytoskeleton inside of the vesicle. The membrane attached BAR domains in the left and in the right panel have the same intrinsic curvature $C_{p}$. 